\title{
How Much Money Should be Paid for a Patient to Isolate During the COVID-19 Outbreak? A Discrete Choice Experiment in Iran
}

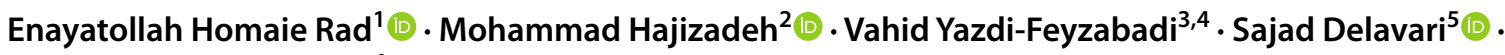 \\ Zahra Mohtasham-Amiri' ${ }^{6}$ iD
}

Accepted: 20 June 2021 / Published online: 27 July 2021

(C) The Author(s), under exclusive licence to Springer Nature Switzerland AG 2021

\begin{abstract}
Introduction Isolation of COVID-19 patients is a vital strategy for preventing the spread of the virus. Isolation without any incentive or compensation for the patients cannot be effective. We sought to find the monetary value of the willingness to accept (WTA) being isolated for COVID-19 in Iran.

Methods In this discrete choice experiment, scenarios were designed by reviewing the literature and semi-structural interviews. Fourteen choice sets with two scenarios were included in an internet-based questionnaire that was sent to the Telegram Social Network. A total of 617 individuals completed the questionnaire. A random-effects logistic regression model was used for the main analysis.

Results The average monetary value of a WTA 7 days of isolation was US\$51.71 (95\% confidence interval [CI] 43.09-60.33). The WTA for one day of isolation was US\$1.48 (95\% CI 1.11-1.85) for unemployed groups, US\$1.49 (95\% CI 1.18-1.79) for office employees and US\$1.36 (95\% CI 0.73-2.01) for manual workers. The WTA was 0.44 (95\% CI 0.35-0.53) US\$ for low-income groups, US\$0.68 (95\% CI 0.52-0.84) for middle-income groups and US\$0.77 (95\% CI 0.35-1.18) for highincome groups.

Conclusions Our findings suggested that financial preferences for being isolated vary widely across individuals within different socioeconomic groups. Policymakers should consider these differences when designing effective intervention to increase compliance with the isolation protocols during infectious disease outbreaks.
\end{abstract}

Vahid Yazdi-Feyzabadi

v_yazdi@kmu.ac.ir

1 Social Determinants of Health Research Center, Guilan University of Medical Sciences, Rasht, Iran

2 School of Health Administration, Dalhousie University, Halifax, Canada

3 Health Services Management Research Center, Institute for Future Studies in Health, Kerman University of Medical Sciences, Kerman, Iran

4 Department of Health Management, Policy and Economics, Faculty of Management and Medical Information Sciences, Kerman University of Medical Sciences, Kerman, Iran

5 Health Human Resources Research Center, Shiraz University of Medical Sciences, Shiraz, Iran

6 Guilan Road Trauma Research Center, Guilan University of Medical Sciences, Rasht, Iran

\section{Key Points for Decision Makers}

The WTA of 7 days of isolation was US\$51.71. The monetary value of WTA increases as days in isolation increases but not in a linear fashion.

Complete payment for all treatment costs was the strongest attribute.

The monetary value of WTA isolation varied across different socioeconomic groups and geographical regions.

\section{Introduction}

Several businesses are facing bankruptcy amid the coronavirus disease 2019 (COVID-19) worldwide pandemic in the past months. This, in turn, has led to high levels 
of unemployment and economic recession. While staying at home, especially for those infected with coronavirus, is an important way to break the virus transmission, some patients do not self-isolate because they may underestimate the dangers of the virus. Some self-employed patients also continue to work because they cannot leave if they are to make ends meet. These groups might affect other members of society $[1,2]$.

Several strategies have been used to control the spread of COVID-19 worldwide. Disinfection of the air in crowded areas by the widespread spray of disinfection substances (e.g., alcohol, cleaning public materials by detergents or alcohol), using personal protection materials (masks and shields), the lockdown of cities and travel restrictions between cities and towns, health promotion methods (promote regular handwashing) and isolation of infected people are the most important ways to control the spread of the COVID-19 in the community. Although all these measures can have a profound impact on reducing new COVID cases, the evidence suggests that isolation of infected people is the most effective way to reduce the spread of infection. If the new cases remain in their homes and self-isolate, the spread of the disease can be managed better [3-5]. Screening for new cases and enforcing the infected cases to stay at home may be impossible without financial and non-financial incentive support [6]. The Chinese government, for example, has awarded prizes to those who visit the Corona Screening Center and test positive for the virus [7].

As a developing country that faces an economic crisis due to the economic sanctions of the USA, Iran is one of the countries that is worst affected by the coronavirus pandemic. Due to the lack of the government budget, Iran was one of the first countries to reduce public interventions to control the spread of COVID-19 infection. For example, the lockdown ended earlier than the time needed for controlling the infection to reduce the overwhelming economic pressure faced by Iranians [8].

Although self-isolation at home or in a safe area is highly recommended, some patients cannot self-isolate because they lack income support. For example, a recent report from the Ministry of Health and Medication (MOHME) in Iran has shown that $20 \%$ of COVID-19-infected patients do not follow the isolation rules [9]. Therefore, it is crucial to know what amount of money would encourage patients to leave their work and stay at home (i.e., their WTA) to design public health strategies for COVID-19 epidemic control. Notwithstanding, there is a lack of information about the WTA isolation for individuals infected with COVID-19. This study, for the first time, is using the discrete choice experiment (DCE) method to identify the amount of money that should be paid for patients to leave their work and stay at home. These findings are essential for policymakers to make the best policies to encourage patients to self-isolate at home.

\section{Methods}

\subsection{Survey Design}

A DCE method, a commonly used method in health economics literature to obtain individuals' preferences and the relative importance of discrete attributes, was used to measure the amount of money required for a patient to isolate. Using this method, participants select attributes of a product or service. Based on their selection, it becomes evident why a particular product or service is preferred over others. In our case, we aimed to investigate the amount of money participants are asking (WTA) for more extended isolation. First, we designed the scenarios through information collected from semi-structured interviews. Interviews were conducted to identify the most important incentives for choosing isolation in Iran. Fourteen interviews were conducted with different expertise and population (e.g., epidemiologists, health economists, social scientists, people who isolate themselves due to the COVID-19 virus, housemakers, and self-employed individuals). A literature review was also performed to find other missed variables. This information was then used to identify the most important attributes of isolation. A focus-group interview was conducted with five researchers each with research backgrounds in epidemiology, health policy, health promotion, and community medicine. The interview asked the researchers to prioritize the attributes and suggest the levels of the most important attributes resulting in the four most important attributes being selected. These attributes included the number of days to be isolated (five levels of $0,7,14,21$ and 30 days), payment (as a percentage of income) for being in isolation (five levels of $0 \%, 25 \%, 50 \%, 75 \%$ and $100 \%$ ), payment (as a percentage of total treatment cost) for COVID-19 treatment (five levels: $0 \%, 25 \%, 50 \%, 75 \%$ and $100 \%$ ), and living services (three levels: no services; food and essential services; food; essential services and entertainment). Table 1 shows the attributes and levels of the attributes.

Two alternatives of attributes were included in each question. A dataset containing the full factorial was identified. Using an orthogonal method, an optimal design of 14 choice sets with two scenarios was identified with a calculated $D$-efficiency of 1.18 . Stata/SE version 13.1 was used for coding and selection of the best choice sets. An example of a choice selection is provided in Table 2 . 
Table 1 Attributes and levels

\begin{tabular}{ll}
\hline Attribute & Level \\
\hline Number of days being isolated & 0 \\
& One week \\
Two weeks & Three weeks \\
One month & 0 \\
Payment for being isolate & One-quarter of household income \\
& Half of household income \\
& Three-quarter of household income \\
& Equal to household income \\
COVID-19 treatment payments & 0 \\
& One-quarter of the total treatment cost \\
& Half of the total treatment cost \\
Three-quarter of the total treatment cost & Total treatment cost \\
Services & No services \\
& Food and essential services \\
& Food and essential services and entertainments
\end{tabular}

Table 2 Example of a discrete choice experiment question

\begin{tabular}{ll}
\hline Choice A & Choice B \\
\hline Two weeks' isolation & Without isolation \\
Payments of three-quarters of the treatment cost & Payments for half of treatment cost \\
Payments for half of household income & Payments for one-quarter of household income \\
Foods, essential services, entertainment & Nothing \\
If you had COVID-19, which one of the two options described would you choose? \\
Choice A O & Choice B O \\
\hline
\end{tabular}

\subsection{Data Collection}

An internet-based survey was designed for data collection. Studies have shown that internet-based surveys are effective enough to be used for DCE studies [10,11]. Questions related to demographic and socioeconomic characteristics of the participants (age, sex, literacy level, income, place of residence, ethnic background, wealth status, education level and working condition) were asked, and between March 2020 to May 2020 the designed scenarios were shared with the potential participants through Telegram social network. As a user-friendly social network, Telegram has 42 million (50\% of Iran's total population) users in Iran. This social network has city- and county-based communities, which can be used for sending online questionnaires. In total, 2488 users saw the questionnaire, and 642 users completed the questions (response rate: $24.8 \%$ ). The average response time to the questions was 8:46 min. We deleted those respondents who completed the questions earlier than the second standard deviation of time (19 respondents) and those who made a mistake in responding to a question with a rational and clear answer (13 respondents). Specifically, we had an additional question in the survey asking respondents to choose from the following two options of receiving either (a) $100 \%$ of household income, $100 \%$ of treatment costs, food, essential services and entertainments or (b) no payment for income loss, no payment for treatment costs, and no services, and 7 days of isolation in both options. We dropped those who selected the second option from the sample. This yielded a final sample of 617 individuals. The Deputy of Research at Kerman University of Medical Sciences approved the study (Ethics No: IR.KMU.REC.1399.369).

\subsection{Statistical Analysis}

The random utility is the theoretical approach for DCE. Due to the random utility, the person $n$ chose $i$ between two alternatives of $i$ and $j$ only if:

$U_{n i}>U_{n j}$

where $U_{n i}$ is the utility of $i . U$ cannot be calculated directly, but it can be compared between different alternatives. If a person chooses $i$ against $j$ this means that $i$ has more utility than $j$. In the present study, different alternatives of isolation were compared together as selecting the stated preferences. The conditional regression model of this study can be written as: 


$$
\begin{aligned}
U_{i}= & \beta_{1} \text { pay }_{i}+\beta_{2} \text { day }_{i}+\beta_{3} \text { day }_{i}+\beta_{4} \text { day }_{i} \\
& +\beta_{5} \text { day }_{i}+\beta_{6} \operatorname{tr} 2_{i}+\beta_{7} \operatorname{tr} 3_{i} \\
& +\beta_{8} \operatorname{tr} 4_{i}+\beta_{9} \operatorname{tr} 5_{i}+\beta_{10} \operatorname{serv} 2_{i} \\
& +\beta_{11} \operatorname{serv} 3_{i}+\varepsilon_{i} .
\end{aligned}
$$

where pay indicated the payments for being isolated, which was analyzed as a continuous form and was calculated by multiplying respondents' household income by the percentage of payments, which was asked in the DCE survey. Day2, Day3, Day4, Day5 were the number of days for being isolated, which was analyzed in dummy form in 4 states of 7 , 14, 21 and 30 days, and the state of 0 days was used as a base category. $\operatorname{tr} 2, \operatorname{tr} 3, \operatorname{tr} 4$, tr 5 were the dummy variables of treatment costs paid when the person faced with COVID-19 in 4 states of $25 \%, 50 \%, 75 \%$, and $100 \%$ and the state of being without treatment payment was used as a reference category. serv2 and serv 3 were the dummy variables of the services provided for being isolated in two states of food plus essential services and food plus essential services plus entertainment, and the state of no services provided was used as a base category. The dependent variable of this model $(U)$ is the expected utility of selecting each alternative. However, as the utility cannot be calculated numerically, it is considered a binary variable $(0=$ not preferring the alternative, 1 $=$ preferring the alternative), demonstrating the utility of the alternative. Data were analyzed using a mixed-effects logistic regression model. The Hausman test suggested that regression analysis with the random-effects is a more appropriate estimator; thus, we did not use fixed-effects models in our estimation. The payment variables in the DCE model were not distributed normally. Non-normal distribution of attributes might lead to bias in WTA estimates in mixedeffects logistic regressions [12]. We first used mixed-effects logisitic regression for the estimation of the models. Since standard deviations obtained from these estimations were not significant, we utilized random-effects logistic regression in the estimations. In addition, WTA was calculated for the variables. WTA is a practical finding for policymakers for pricing of the isolation. We utilized the random-effects logistic regression results for calculating WTA. WTA for the number of days being isolated can be calculated using the formula below, which demonstrates the monetary value of one day of isolation:

$\mathrm{WTA}(\mathrm{pl})=-\frac{\sigma U / \text { days }}{\sigma U / \sigma \text { pay }}=-\frac{\beta_{3}}{\beta_{1}}$.

Similarly, WTA can also be calculated for other attributes. Due to potential heterogeneity in different socioeconomic, wealth and health condition groups, we performed our analysis for different groups, separately. The exchange rate for converting IR.Rials to US\$ was IR.Rials $125,000=$ US $\$ 1$ at the time of the study. All analyses were performed using STATA SE software version 13.1.

\section{Results}

\subsection{Sample Characteristics}

Of a total 617 participants, 369 (59.81\%) were females, 89 $(14.43 \%)$ had less than primary school degrees, 46 (7.46\%) had secondary school education, $242(39.22 \%)$ had high school education, and the remaining 240 (39.9\%) had a university degree. According to the most recent Iranian population census, $29 \%$ of the Iranian population had primary education, $18 \%$ had a secondary education degree, $30 \%$ had a high school degree, and $22 \%$ had a university degree [13], suggesting that our study sample is approximately similar to the Iranian population. The majority of participants $(263,42.63 \%)$ were white-collar workers, 159 (25.77\%) participants were not in the labor force (e.g., students, housemakers, retired), $38(6.16 \%)$ were unemployed, 21 (3.40\%) were manual workers, and 72 (11.67\%) were self-employed. The average age of participants was $35.87 \pm 0.40$ years, and the average monthly income of the household was US $\$ 45.30 \pm 0.877$ ( 1 US $\$=125,000$ IR.Rial at the time of this study).

Table 3 shows the self-perceived health status, economic conditions, and health priority of the participants, which was collected using the visual analogue scale (VAS) forms. The participants selected a number between 1 and
Table 3 Health status, economic condition and health priority of participants

\begin{tabular}{llll}
\hline Variable & Mean & Standard error & $\begin{array}{l}95 \% \\
\text { confidence } \\
\text { interval }\end{array}$ \\
\hline $\begin{array}{l}\text { Self-perceived health: rating on a scale 1 (poor)_-10 } \\
\quad \text { (excellent) }\end{array}$ & 7.81 & 0.07 & $7.66-7.95$ \\
$\begin{array}{l}\text { Self-perceived economic condition: rating on a scale 1 } \\
\text { (low)—10 (high) }\end{array}$ & 4.82 & 0.07 & $4.68-4.96$ \\
Health priority: rating on a scale 1 (low)—10 (high) & 9.30 & 0.05 & $9.19-9.40$ \\
\hline
\end{tabular}


10 to describe their health, economic condition and health priority compared to other services/goods. As reported in the table, the average self-perceived health status was 7.81 (95\% confidence interval [CI] 7.66-7.95), and economic condition status was 4.82 (95\% C: $4.68-4.96)$. The average health priority of participants was 9.298 (95\% CI 9.19-9.40).

\subsection{Regression Results}

Table 4 shows the results of random-effects logistic regression. We used payments for being isolated in a continuous form and other variables as dummy variables. As shown in Table 4, people preferred higher payments for being isolated (coefficient $=0.02,95 \%$ CI 0.02-0.02), a higher number of days being isolated (coefficient of 30 days being isolated $=$ $1.18,95 \%$ CI 1.06-1.29; coefficient of 7 days being isolated $=0.94,95 \%$ CI 0.83-1.05) when they are facing COVID- 19 . In addition, individuals' preference for the second service package, i.e., food and essential services (coefficient $=0.83$, 95\% CI 0.75-0.92) was higher than the third package, i.e., food, essential services and entertainments (coefficient $=$ $0.67,95 \%$ CI $0.59-0.75$ ). As a rational selection, they prefer higher percentage of COVID-19 treatment cost (coefficient on $100 \%$ coverage of treatment $=0.80,95 \%$ CI $0.69-0.91$; coefficient on $75 \%$ coverage of treatment cost coefficient $=0.79,95 \%$ CI $0.69-0.90$; coefficient on $25 \%$ coverage of treatment cost coefficient $=0.27,95 \%$ CI $0.15-0.39$ ).
Table 5 contains the results of random-effects logistic regression of the selected choices and the monetary value of WTA in IR. Rials and US\$ generated using the marginal rate of substitution. The WTA estimates were negative and significant at $95 \% \mathrm{CI}$, indicating that people prefer to receive money for being isolated. Thus, the absolute values of Willingness to Pay (WTP) results can be considered as the willingness to accept (WTA). From the attributes, the strongest is payment for 30 days of isolation, and participants preferred that more than others (WTA $=$ US $\$ 64.45 \pm 4.94$ ). The WTA for 21 days of isolation was US\$56.37 ( \pm 4.61 ), for 14 days of isolation was US\$45.75 $( \pm 4.09)$, and for 7 days of isolation was US\$51.71 ( \pm 4.40$)$. When we considered the days of isolation as a continuous form, the average WTA for one day of isolation is US\$1.58 ( \pm 0.12$)$ (estimated in another model and not included in the table). The WTA estimates for the treatment costs coverage was US $\$ 14.85$ ( \pm $3.26)$ for $25 \%$ coverage of treatment cost, US $\$ 47.23( \pm 3.72)$ for $50 \%$ coverage of treatment cost, US $\$ 43.54( \pm 3.70)$ for $75 \%$ of treatment cost coverage and US $\$ 43.97( \pm 3.75)$ for total coverage of treatment costs. In addition, the WTA for (1) food and essential services and (2) for food, essential services and entertainments were US\$45.65 $( \pm 3.45)$ and US\$36.50 ( \pm 3.17 ), respectively.

Table 6 presents the monetary value of WTA for 1 day of isolation in different socioeconomic groups. All of the models were analyzed using random-effects logistic regression. Number of days was used as a continuous
Table 4 Results of randomeffects logistic model with payment as a continuous variable

\begin{tabular}{llll}
\hline Choice & Coefficient & Standard error & 95\% confidence interval \\
\hline $\begin{array}{l}\text { Payment for isolation (pay) } \\
\text { Without isolation (Ref.) }\end{array}$ & 0.02 & 0.00 & $0.02-0.02$ \\
Isolation for & & & \\
7 days & 0.94 & 0.06 & $0.83-1.05$ \\
14 days & 0.83 & 0.06 & $0.72-0.95$ \\
21 days & 1.03 & 0.06 & $0.92-1.14$ \\
30 days & 1.18 & 0.06 & $1.06-1.29$ \\
Without treatment payment (Ref.) & & & \\
Percentage of treatment cost & & & \\
$\quad 25 \%$ & 0.27 & 0.06 & $0.15-0.39$ \\
$\quad 50 \%$ & 0.86 & 0.06 & $0.75-0.97$ \\
$\quad 75 \%$ & 0.79 & 0.05 & $0.69-0.90$ \\
$\quad 100 \%$ & 0.80 & 0.06 & $0.69-0.91$ \\
No services (Ref.) & & & \\
Food and essential services & 0.83 & 0.04 & $0.75-0.92$ \\
Food, essential services and entertainments & 0.67 & 0.04 & $0.59-0.75$ \\
Constant variable & -2.17 & 0.06 & -2.30 to -2.05 \\
Sigma-u & 0.0002 & 0.00313 & \\
$P$ & 0.000 & 0.000 & \\
Log-likelihood & $-10,925$ & & \\
\hline
\end{tabular}

Ref. indicates the reference category in the regression 
Table 5 The random-effects logistic regression results of the selected choices and the monetary value of willingness to accept (WTA)

\begin{tabular}{|c|c|c|c|c|}
\hline Choice & $\begin{array}{l}\text { Willingness to accept } \\
\text { (WTA in \$US) }\end{array}$ & Standard error & $\begin{array}{l}\text { Willingness to accept } \\
\text { (WTA in IR.Rials) }\end{array}$ & Standard error \\
\hline \multicolumn{5}{|l|}{ Without isolation (Ref.) } \\
\hline 7 days of isolation & -51.71 & 4.40 & $-6,463,979$ & 549,864 \\
\hline 14 days of isolation & -45.75 & 4.09 & $-5,718,245$ & 511,670 \\
\hline 21 days of isolation & -56.37 & 4.61 & $-7,046,491$ & 576,299 \\
\hline 30 days of isolation & -64.45 & 4.94 & $-8,055,880$ & 617,409 \\
\hline \multicolumn{5}{|l|}{ Without treatment payment (Ref.) } \\
\hline Payment of $25 \%$ of the treatment cost & -14.85 & 3.26 & $-1,856,104$ & 407,927 \\
\hline Payment of $50 \%$ of the treatment cost & -47.23 & 3.72 & $-5,903,148$ & 465,178 \\
\hline Payment of $75 \%$ of the treatment cost & -43.54 & 3.70 & $-5,442,440$ & 462,353 \\
\hline Payment of $100 \%$ of the treatment cost & -43.97 & 3.75 & $-5,496,325$ & 468,366 \\
\hline \multicolumn{5}{|l|}{ No services (Ref.) } \\
\hline Food and essential services & -45.65 & 3.45 & $-5,706,211$ & 431,673 \\
\hline Food, essential services and entertainments & -36.50 & 3.17 & $-4,562,981$ & 395,797 \\
\hline
\end{tabular}

Ref. indicates the reference category in the regression. All values were statistically significant at a $95 \%$ confidence interval. Exchange rate: 1 US $\$=125,000$ IR.Rials

Table 6 Willingness to accept (WTA) for one-day isolation in different socioeconomic groups

\begin{tabular}{|c|c|c|c|c|c|}
\hline & \multirow[t]{2}{*}{$n$} & \multicolumn{2}{|l|}{ IR.Rials } & \multicolumn{2}{|l|}{ US\$ } \\
\hline & & WTA & Standard error & WTA & Standard error \\
\hline \multicolumn{6}{|l|}{ Occupation status } \\
\hline Unemployed-out of the labor force & 5516 & $-185,112$ & 23,489 & -1.48 & 0.19 \\
\hline White-collar workers & 3248 & $-186,026$ & 19,368 & -1.49 & 0.15 \\
\hline Manual workers & 5908 & $-170,051$ & 39,895 & -1.36 & 0.32 \\
\hline \multicolumn{6}{|l|}{ Self-perceived health status } \\
\hline Poor (scales 1-3) & 532 & $-202,964$ & 90,389 & -1.62 & 0.72 \\
\hline Good (scales 4-7) & 5404 & $-145,620$ & 28,031 & -1.16 & 0.22 \\
\hline Excellent (scales 8-10) & 11,340 & $-207,527$ & 17,621 & -1.66 & 0.14 \\
\hline \multicolumn{6}{|l|}{ Education status } \\
\hline Non-academic & 3780 & $-156,757$ & 26,557 & -1.25 & 0.21 \\
\hline Academic & 13,496 & $-195,940$ & 16,741 & -1.57 & 0.13 \\
\hline \multicolumn{6}{|l|}{ Health priority } \\
\hline Low (scales 1-3) & 280 & $-47,797$ & 54,546 & -0.38 & 0.44 \\
\hline Medium (scales 4-7) & 1120 & $-211,372$ & 82,033 & -1.69 & 0.66 \\
\hline High (scales 8-10) & 15,876 & $-197,474$ & 15,386 & -1.58 & 0.12 \\
\hline \multicolumn{6}{|l|}{ Income } \\
\hline Low (scales 1-3) & 6860 & $-55,203$ & 5,777 & -0.44 & 0.05 \\
\hline Medium (scales 4-7) & 6272 & $-84,748$ & 10,119 & -0.68 & 0.08 \\
\hline High (scales 8-10) & 4144 & $-96,125$ & 26,443 & -0.77 & 0.21 \\
\hline \multicolumn{6}{|l|}{ Wealth groups } \\
\hline 1st quintile & 1232 & $-238,001$ & 123,399 & -1.90 & 0.99 \\
\hline 2nd quintile & 6188 & $-137,663$ & 16,335 & -1.10 & 0.13 \\
\hline 3rd quintile & 6888 & $-140,156$ & 16,043 & -1.12 & 0.13 \\
\hline 4th quintile & 2548 & $-216,819$ & 46,364 & -1.73 & 0.37 \\
\hline 5th quintile & 420 & $-396,163$ & 209,936 & -3.17 & 1.68 \\
\hline
\end{tabular}


variable and other variables as dummies in the estimations. As reported in the table, the unemployed and participants who are not in labor force (students, housemakers, retirees) are willing to receive US\$1.48 (95\% CI 1.11-1.85) to accept being isolated, while white-collar workers and manual workers are willing to receive US\$1.49 (95\% CI 1.18-1.79) and US\$ 1.36 (95\% CI 0.73-2.01), respectively, to accept one-day isolation. The WTA one day of isolation in those participants with poorly perceived health status was US $\$ 1.62(95 \%$ CI 0.21-3.04), while for those with excellent perceived health status, the WTA was US\$1.66 (95\% CI 1.38-1.94). Participants without academic degrees were willing to accept US\$1.25 (95\% CI 0.84-1.67) to be isolated for one day, whereas those with academic degrees were willing to accept US\$1.57 (95\% CI 1.3-1.83) for one day of isolation. Participants giving low priority to health in their life were willing to accept US $\$ 0.38$ (95\% CI -0.47 to 1.23 ) for one-day of isolation, and those rating health as a medium and a high priority in their lives were WTA US\$1.69 (95\% CI 0.40-2.98) and US\$1.58 (95\% CI 1.34-1.82), respectively, for one day of isolation. The momentary value of WTA for one day of isolation for low, middle, and high income was US\$0.34 (95\% CI $0.23-0.44)$, US $\$ 0.57$ (95\% CI $0.46-0.68$ ) and US $\$ 0.95$ (95\% CI 0.60-1.3), respectively.

Figure 1 demonstrates the WTA for being isolated for one day among different provinces of Iran. As shown in the figure, participants living in the western provinces of the country had the highest monetary value of WTA for one day of isolation, while participant from south-western provinces had the lowest monetary value of WTA for one day of isolation (WTP value for eastern provinces was the highest but it was not significant at 95\% CI). Figure 2 shows the WTA for being isolated for one day in five wealth quintile groups. As illustrated in the figure, the WTA of the wealthiest quintile group was higher than other wealth quintile groups.

\section{Discussion}

Appropriate public health interventions at different stages of an infectious disease outbreak are of paramount importance. Some public health interventions, such as isolation of infected and exposed individuals, effectively control infectious diseases (e.g., SARS) besides pre-emptive vaccination $[14,15]$. Moreover, ample evidence suggests that isolation and quarantine policies effectively contained the COVID19 outbreak [16-19]. However, little is known about public preferences for these interventions or how they trade off different intervention levels. One major obstacle to compliance and adherence to self-isolation and quarantine scenarios is the loss of income during isolation. This study used a DCE approach that relied on people's preferences to indirectly measure the amount of money that people would accept to stay at home when they were infected during the COVID-19 outbreak.

Results suggested that people prefer higher coverage for treatment of COVID-19 as an option for being isolated, and they prefer foods, essential services and entertainment besides the isolation with a higher WTA in favor of foods
Fig. 1 Monetary value (US\$) of willingness to accept for being isolated for one day among different provinces of Iran

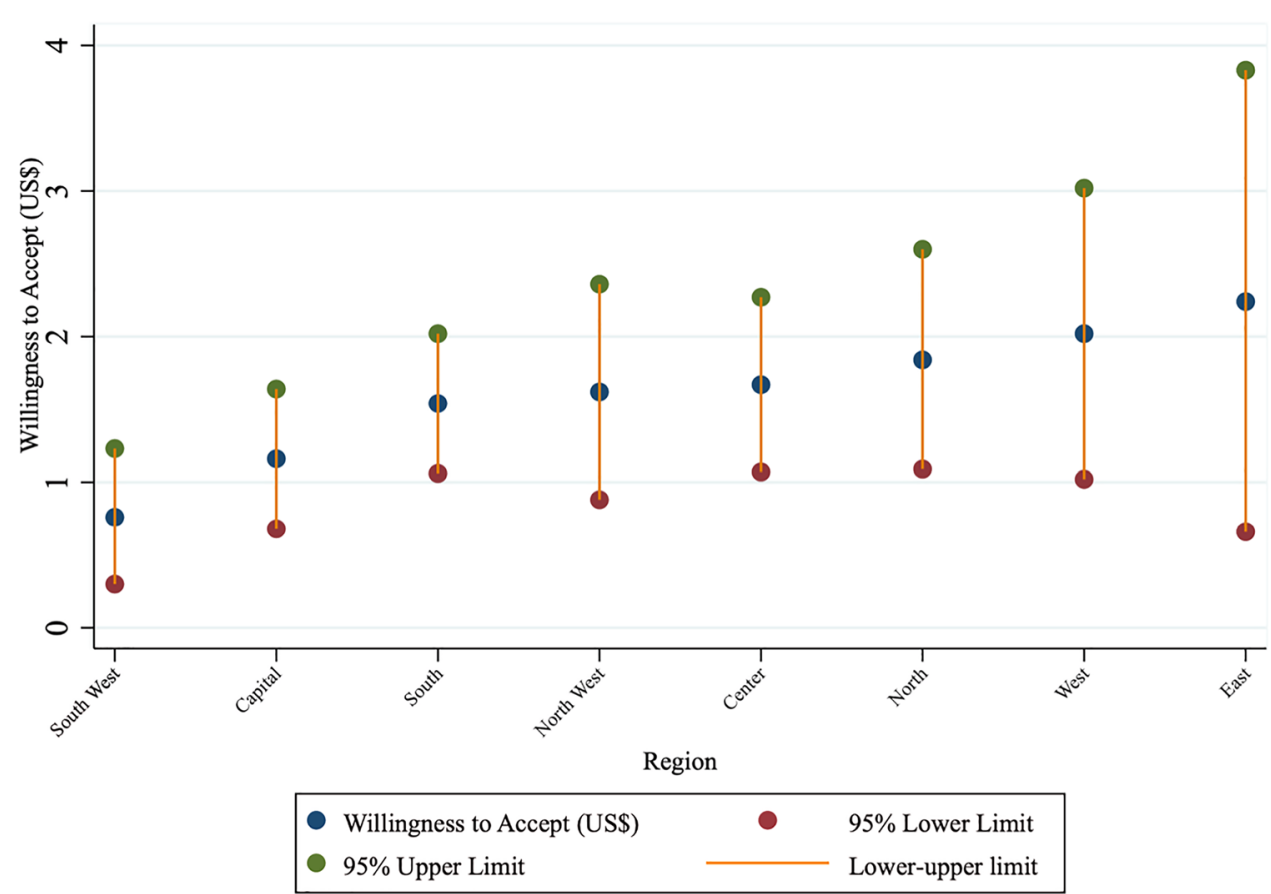


Fig. 2 Monetary value (US\$) of willingness to accept for being isolated for one day among five income quintile groups in Iran

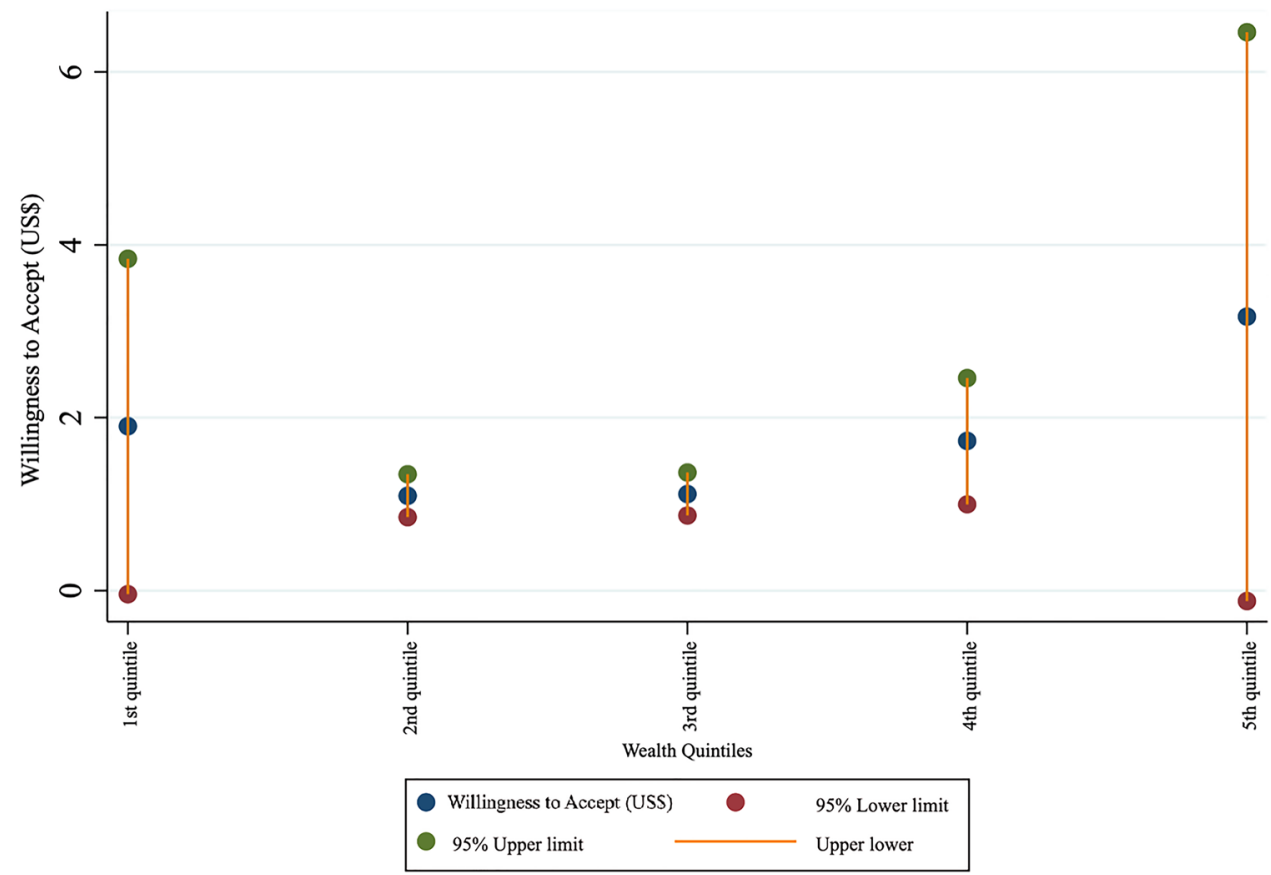

and essential services. To encourage compliance with quarantine, a study conducted in the USA by Rothstein et al [20] proposed some options such as guaranteed job security and provision of income replacement (e.g., financial assistance program, employment insurance regulations) for different targeted groups including patients, health-care workers, unemployed people, and people with interrupted employment. A study in Canada showed that lack of logistic support such as delivering groceries and providing other services necessary for daily living and medical supplies, affected compliance with quarantine during the SARS outbreak [21].

We concluded that the monetary value of WTA being isolated varied across different socioeconomic groups. These findings can help policymakers understand WTA for isolation among different population segments, which is essential for making informed resource allocation to increase compliance with the isolation protocols during infectious-disease outbreaks [22]. Individuals belonging to higher compared to lower social class occupations (e.g., white-collar workers) were willing to accept the isolation and quarantine for more money. This can be due to the fact that individuals with higher social class jobs have higher income and, therefore, higher opportunity costs for isolation. Another important finding was that low- compared to high-income individuals requested less money to go into isolation. This result suggests an income sensitivity (i.e., differential WTA for isolation according to income level) in self-isolation. Occupations in Iran reflect the social status of the individuals. While labor and manual working jobs are considered lowlevel jobs, management and businesses occupations belong to high-level jobs. On this basis, wages and salaries paid are a function of the job identity in terms of specialization, complexity, prestige, and level of education needed. For this reason, jobs with higher wages naturally demand higher compensation when isolation is necessary. This is due to the higher daily value of wages for days of absence from work.

The monetary value of WTA being isolated was found to be different across educational groups. People with academic education degrees are willing to be isolated if they receive more than those with non-academic education. A study conducted in the USA showed that high school graduates or high school plus technical school graduates had a higher likelihood of compliance with work-related pandemic influenza isolation strategies than people with a college education level [23]. A possible explanation for this might be that people with higher education level during an emerging infectious disease suffer more losses and thus prefer to receive more compensation for losses than those with lower educational attainments.

Another factor affecting people's monetary value of WTA for being isolated is prioritizing health in their lives. We found people who choose their health as a low priority tend to be isolated by receiving less money. Results also showed that people with higher self-rated health status are willing to be isolated if they receive more money than those with lower self-rated health status.

There is a difference across provinces in terms of the amount of WTA for being isolated in Iran. The main aim of looking at the different regions of Iran was to provide clear information to the policymakers about regional variations of WTA for being isolated. This information can help policymakers implement region-specific, instead of 
uniform, policies to encourage people to self-isolate during the pandemic. Results revealed that participants living in the western regions of Iran tend to accept self-isolated if they receive more money than those living in south-western regions. Although there is some evidence to support the association between geographical and racial differences and treatment preferences of patients $[24,25]$, we could not find evidence to support the association in the context of emerging infectious diseases. People living in western regions in Iran belong to ethnicities different from south-western region and have different cultural backgrounds. A possible explanation for this might be that different ethnicities have different preferences regarding health beliefs, perceptions of the benefit of isolation and quarantine in preventing the disease, and perceived risk of the outbreak [26, 27]. Another possible explanation could be the differences in development levels (e.g., economic and employment infrastructures) among Iranian provinces [28]. Further research can help to identify factors that account for the geographical differences in WTA for isolation in Iran.

Results of a review about how to improve adherence to quarantine during an infectious disease outbreak showed that the government should ensure the provision of sufficient supplies of food, medication, and other essentials [29]. Two recent studies conducted in Australia [30] and Canada [21] showed that supportive measures (e.g., the continuation of wages, salaries, and other forms of income while they were not working and in quarantine) could be helpful to stimulate public participation in pandemic. A cohort study conducted in Israel in two time points (February and March 2020) during the COVID-19 outbreak concluded that providing people with assurance about their livelihood during self-quarantine is an important component of compliance with self-isolation [31, 32].

Another study conducted by Blake et al in the USA [23] showed that lack of paid sick leave and income affect adherence to the recommended isolation and quarantine guidelines and should be considered the primary target for workplace interventions during an outbreak. Several studies also found that the requirement to attend work and fear of loss of income and loss of employment after the quarantine as reasons for not adhering to quarantine protocols [20, 21, 33].

Some limited studies have investigated the WTA quarantine during infectious disease outbreaks. A study by Cook et al in 2018 investigated the peoples' preferences for government interventions during an outbreak. They found that people prefer interventions that result in lower deaths and taxes [34]. In a study, Himmler et al calculated willingness to pay for an early-warning system for infectious diseases in some European countries and found that the willingness to pay for such a system was US $\$ 21.80$.

This study indicated that government financial support could enhance people's willingness to stay at home and comply with isolation. The financial support may show government credibility in helping people to adhere to the prevention and control measures against COVID-19 and thus, enhance their willingness to be isolated. More government credibility has been shown to increase the willingness to quarantine [35]. Our result suggested that in a developing country such as Iran, which faces economic instability, staying at home can jeopardize the household's stability. Different socioeconomic groups have different monetary values of WTA for being isolated; thus, effective intervention efforts to increase compliance with the isolation protocols during infectious disease outbreaks should vary across different socioeconomic groups.

\section{Suggestions for Policymakers}

During public health emergencies or emerging infectious diseases such as the COVID-19 epidemic, self-isolation is a vital measure to control and contain epidemics and flatten the epidemic curves. This study has some important practical implication for policymakers. First, in the face of communicable diseases, particularly those with high contagion, there is a definite need to consider public preferences to increase public compliance with social interventions such as self-isolation and business closure. Second, Iran's government should financially compensate, support, and provide essential daily services for people infected or suspected of having the disease during the quarantine period to increase self-isolation and consequently help contain the epidemic. Third, when applying supportive policies such as social and financial assistance, these policies should be tailored differently across demographic features such as education level, socioeconomic factors such as income level and job classes.

The results of this study showed that an individual in Iran is willing to accept US\$1.58 and US\$64.45 for being isolated for one day and one month, respectively. The isolation could be effective if all household members (in addition to the infected one) follow the isolation rules. Therefore, to have effective isolation, payments must be made that take into account the household size. This can be calculated using the consumption equivalence scale, which uses the relationship between total household consumption and household size [36]. Additionally, the risk of inaccurate reporting of COVID-19 infection with the purpose of earning money is inevitable. Governments can use official testing organizations or trustworthy laboratories for confirmation of infection with COVID-19. Online reporting systems can be effective to avoid crowding at the time of registering for the payments. Based on our results, the amount of compensation given for job losses can vary per length of forced isolation, and the relationship between days of being isolated and 
the amount of compensation (US\$) does not follow a linear relationship.

\section{Strengths and Limitations of the Study}

This study is one of the first to measure the preferences of the general public regarding isolation intervention. The results can be used to form part of the established preparedness plan. Another strength of this study was the subgroup analyses for some socio-cultural features such as self-reported health priority and self-perceived health status. These analyses provided appropriate evidence for health policymakers to understand how isolation acceptability and compliance are complex and diverse among different populations. The main limitation of our study is that few preferences (e.g. providing and delivering foods and essential services door-to-door) are stated, and they are based on less realistic hypothetical conditions, which are not easy to implement in Iran. Therefore, it is not known to what extent the reported preferences during an actual pandemic vary from the actual preferences. This limitation may affect the preference of respondents to select paired scenarios.

\section{Conclusion}

Financial incentives or supports are important strategies in persuading people to choose isolation and stay at home to prevent the transmission of COVID-19. To provide these financial incentives, we should be aware that financial preferences for being isolated vary widely across individuals with different socioeconomic, health, and demographic status. Financial incentives must also cover all household members in order to have effective isolation in the prevention of spreading COVID-19.

\section{Declarations}

Funding This project received no financial support.

Conflicts of interest/Competing interests The authors declare that they have no conflicts of interest.

Ethics approval The Ethics Committee of Kerman University of Medical Sciences (IR.KMU.REC.1399.369) approved this study. Ethical considerations were taken into account during data collection and analysis. The confidentiality of the participants was protected, and no personal information could be identified in any publications arising from the study. Participants were informed that their participation is entirely voluntary.

Consent to participate A question indicating written informed consent was obtained from the participants before starting the questions of the study.
Consent for publication Not applicable.

Availability of data and material The data used in the study are available from the corresponding author on reasonable request.

Code availability We used STATA SE software version 13.1 codes for our analyses. The codes are available on the STATA Corp website.

Authors' contributions EHR participated in designing the study, gathering data, analyzing and interpreting data, and writing the manuscript. VYF participated in designing the study, assisted in analyzing and interpreting the data, and writing the manuscript. MH critically revised the manuscript several times and had several intellectual comments on the drafted manuscript. SD provided advice in the study's implementation, the data collection, and assisted in preparing the manuscript. ZMA assisted in the drafting of the questionnaire and the data collection. All authors read and approved the final version of the article.

\section{References}

1. Lebas B, et al. Control and monitoring: quarantine situation of Plum pox virus in New Zealand. EPPO Bull. 2006;36(2):296-301.

2. Holshue ML, et al. Washington State 2019-nCoV Case Investigation Team. First Case of 2019 Novel Coronavirus in the United States. N Engl J Med. 2020;382(10):929-36.

3. Kucharski AJ, et al. Early dynamics of transmission and control of COVID-19: a mathematical modelling study. Lancet Infect Dis. 2020;20:553-8.

4. Li Y, Xia L. Coronavirus disease (COVID-19): role of chest CT in diagnosis and management. Am J Roentgenol. 2020;214:1-7.

5. Organization WH. Considerations for quarantine of individuals in the context of containment for coronavirus disease (COVID-19): interim guidance. World Health Organization; 2020.

6. Haddix AC, Teutsch SM, Corso PS. Prevention effectiveness: a guide to decision analysis and economic evaluation. Oxford University Press; 2003.

7. Kelly E. China was slammed for initial COVID-19 secrecy, but its scientists led the way in tackling the virus [Internet]. NewYork. 2020 Apr 7. Available from: https://sciencebusiness.net/covid-19/ international-news/china-was-slammed-initial-covid-19-secrecyits-scientists-led-way

8. Sadeghi S. Why Hassan Rouhani Ended Iran's lockdown. [Internet], Foreign Policy, Washington. 2020 May 5. Available form: https://foreignpolicy.com/2020/05/05/why-hassan-rouhani-endedirans-lockdown

9. Harirchi I. Corona mobile bombs in the city; $20 \%$ of COVID-19 patients are not quarantined!, in Hamshahri Newpaper. Hamshahrionline; 2020.

10. Ryan M, Gerard K, Amaya-Amaya M. Using discrete choice experiments to value health and health care, vol. 11. Springer; 2007.

11. Determann D, et al. Impact of survey administration mode on the results of a health-related discrete choice experiment: online and paper comparison. Value Health. 2017;20(7):953-60.

12. Regier DA, et al. Bayesian and classical estimation of mixed logit: an application to genetic testing. J Health Econ. 2009;28(3):598-610.

13. Center IS. Iranian population census. 1st ed. Tehran: ISC; 2016.

14. Control CfD, et al. Quarantine and isolation: lessons learned from SARS. University of Louisville School of Medicine; 2003. 
15. Alam M, Kabir KA, Tanimoto J. Based on mathematical epidemiology and evolutionary game theory, which is more effective: quarantine or isolation policy? J Stat Mech Theory Exp. 2020;2020(3): 033502.

16. Huang $\mathrm{Y}, \mathrm{Wu} \mathrm{Y}, \mathrm{Zhang} \mathrm{W}$. Comprehensive identification and isolation policies have effectively suppressed the spread of COVID19. Chaos Solitons Fractals. 2020;139: 110041

17. Wilder-Smith A, Freedman DO. Isolation, quarantine, social distancing and community containment: pivotal role for old-style public health measures in the novel coronavirus $(2019-n \mathrm{CoV})$ outbreak. J Travel Med. 2020;27(2):taaa020.

18. Sjödin $\mathrm{H}$, et al. Only strict quarantine measures can curb the coronavirus disease (COVID-19) outbreak in Italy, 2020. Eurosurveillance. 2020;25(13):2000280.

19. Wilasang C, et al. Reduction in effective reproduction number of COVID-19 is higher in countries employing active case detection with prompt isolation. J Travel Med. 2020;27:taaa095.

20. Rothstein MA, Talbott MK. Encouraging compliance with quarantine: a proposal to provide job security and income replacement. Am J Public Health. 2007;97(Supplement_1):S49-56.

21. DiGiovanni $\mathrm{C}$, et al. Factors influencing compliance with quarantine in Toronto during the 2003 SARS outbreak. Biosecur Bioterror. 2004;2(4):265-72.

22. Deal K. Segmenting patients and physicians using preferences from discrete choice experiments. Patient Patient Cent Outcomes Res. 2014;7(1):5-21.

23. Blake KD, Blendon RJ, Viswanath K. Employment and compliance with pandemic influenza mitigation recommendations. Emerg Infect Dis. 2010;16(2):212.

24. Constantinescu F, et al. Racial disparities in treatment preferences for rheumatoid arthritis. Med Care. 2009;47(3):350-5.

25. Allaire BT, Brown DS, Wiener JM. Who wants long-term care insurance? A stated preference survey of attitudes, beliefs, and characteristics. INQUIRY. 2016;53(1):1-8.
26. Nikfarjam A, et al. The frequency of alcohol use in iranian urban population: the results of a national network scale up survey. Int J Health Policy Manag. 2017;6(2):97-102.

27. Rad EH, et al. Cigarette smoking and its financial burden among Iranian households: evidence from household income and expenditures survey. J Res Health Sci. 2020;20(4):e00494.

28. Central Bank of Iran. Unemployment rate in Iranian Provinces. 1st ed. Tehran: Central Bank of Iran; 2019.

29. Webster RK, et al. How to improve adherence with quarantine: rapid review of the evidence. Public Health. 2020;182:163-9.

30. Braunack-Mayer AJ, et al. Including the public in pandemic planning: a deliberative approach. BMC Public Health. 2010;10(1):501.

31. Bodas M, Peleg K. Income assurances are a crucial factor in determining public compliance with self-isolation regulations during the COVID-19 outbreak-cohort study in Israel. Israel J Health Policy Res. 2020;9(1):54.

32. Bodas M, Peleg K. Self-isolation compliance in the COVID-19 era influenced by compensation: findings from a recent survey in Israel. Health Aff (Millwood). 2020;39(6):936-41.

33. Teh $\mathrm{B}$, et al. Impact of swine influenza and quarantine measures on patients and households during the H1N1/09 pandemic. Scand J Infect Dis. 2012;44(4):289-96.

34. Cook AR, et al. Public preferences for interventions to prevent emerging infectious disease threats: a discrete choice experiment. BMJ Open. 2018;8(2):e017355-e017355.

35. Xiang P, Guo J, Liu Q. How government credibility and social morality work in a public health emergency. A study of public quarantine willingness in COVID-19. Anal Soc Issues. Public Policy. 2020;20:443-61.

36. $\mathrm{Xu} \mathrm{K}$, et al. Household catastrophic health expenditure: a multicountry analysis. Lancet. 2003;362(9378):111-7. 